\title{
Pathomorphological changes in the testes of males with babesiosis
}

\author{
Viktor Mikhailenko ${ }^{1}$, Olga Dilekova ${ }^{1}$, Vladimir Meshcheryakov ${ }^{1,{ }^{*}}$, Dmitry Chervyakov$^{1}$, \\ Daniil Proskurin ${ }^{1}$ \\ ${ }^{1}$ Stavropol State Agrarian University, 12, Zootekhnicheskiy per., 355017, Stavropol, Russia
}

\begin{abstract}
The aim of the work was to study pathomorphological changes in the testes in males with babesiosis. Diagnosis of babesiosis in sick males was performed based on the detection of typical clinical signs and blood smears. Blood smears were fixed in methanol and stained according to the Romanovsky-Giemsa method with hematoxylin and eosin. For histological studies, pieces of testicles were selected, which were fixed in an $8 \%$ neutral aqueous formalin solution. The prepared material was poured into paraffin according to the conventional method. Histological sections were stained with hematoxylin and eosin using the method of Van Gieson and Mallory. Pathomorphological examination of stained histological sections revealed changes characteristic of parenchymal orchitis. The spermatogenic epithelium of the convoluted seminal tubules was drained down in all fields of vision. The lumen of the tubules was filled with protein detritus, exfoliated cells of the spermatogenic epithelium and macrophages. Part of the Sertoli cells was in a state of vacuolar degeneration. Focal clusters of lymphoid-macrophage infiltrates were visible between the convoluted seminal tubules, especially multiple ones around the blood vessels. Endocrine cells were subjected to atrophy due to compression by cell infiltrates and connective tissue growths.
\end{abstract}

\section{Introduction}

Dog breeding is an integral part of human life, since dogs have long been used in ensuring the safety of housing, protecting herds, in security service, the army, and as companion animals. Currently an important task is to reduce losses from the death of dogs and improve their health. Babesiosis - one of the diseases that lead to the death of dogs, and reduce their reproductive ability. Babesiosis is a natural focal disease, because in the wild fauna there are a large number of susceptible animals, long-term parasitosis and the presence of ticks-carriers. The main carriers of babesias are ticks [1,2].

According to many authors, ticks that carry babesiosis are widespread in many countries $[3,4,5]$. The North Caucasus region is permanently dysfunctional for babesiosis of dogs, which coincides with the habitat of the tick Dermacentor marginatus.

According to our observations, the incidence of babesiosis has a negative effect on the reproductive function of animals. Reduction of sexual activity in males in spring and

\footnotetext{
*Corresponding author: meva26@inbox.ru
} 
autumn period is one of the characteristic clinical signs of babesiosis. In autoimmune orchitis, the quantity and quality of sperm decreases and sexual activity worsens $[6,7,8]$. In the available literature, we found no data on the study of pathomorphological changes in the testes of males with babesiosis, which was the purpose of our research.

\section{Research materials and methods}

The research was conducted in the period from 2010 to 2019 in veterinary clinics of the North Caucasus cities (Stavropol, Pyatigorsk, Budennovsk, Mikhailovsk, Cherkessk) and in the conditions of the Department of Parasitology and Veterinary Expertise, Anatomy and Pathanatomy named after professor S. N. Nikolsky of the Stavropol State Agrarian University. For histological studies, pieces of testes of $0.5 \mathrm{~cm}$ thick were selected from fallen mature males who were diagnosed with babesiosis during their life. A total 14 male cadavers were examined, including five 1-1,5 years-old with an acute course of the disease; six 2-4 years-old animals with an acute course and three 3-6 years-old with a chronic course of the disease. The material was taken no later than 3 hours after the animal death. The diagnosis of babesiosis in sick males was carried out taking into account the characteristic clinical signs and studies of peripheral blood smears taken from sick animals during life. Blood smears were taken from the ear vein. After they have been fixed in methanol they were stained using the Romanovsky-Gimza method and hematoxylin and eosin [9]. In each smear, 100 fields of view were studied under the immersion system of a biological microscope at an x1000 zoom.

To analyze the pathogenic effect of babesias on the testicular tissues a pathoanatomic autopsy was performed on animals that had fallen from babesiosis. During the autopsy, pieces of testes up to $0.5 \mathrm{~cm}$ thick were cut from each cadaver, which were fixed in an $8 \%$ aqueous solution of formalin. Then the material was washed for 24 hours with running water, and after it was dehydrated in ethyl alcohol with increased concentration and poured into paraffin according to the generally accepted method. Obtained paraffin blocks were fixed on wooden blocks. Histological sections with a thickness of 3-5 microns were made on a rotary microtome. Then the sections were stained with hematoxylin and eosin. Hematoxylin was made using the Ehrlich method. To determine the connective tissue elements, the sections were stained according to van Gieson and Mallory. Cross section microscopy was performed using an Olympus BX45 light microscope with a built-in camera.

\section{Results}

In a clinical study of sick animals with acute and superacute babesiosis, we have observed a sharp increase in body temperature to 41.0-41.5 $\mathrm{C}^{\circ}$, depression, refusal of food, lethargy, tachycardia, anemia of the external mucous membranes. The hyperacute course was observed mainly in animals under the age of one year. The mortality rate in the super-acute course of babesiosis, without providing urgent specific treatment, reached 70,0-100,0\%. In the first day after the detection of clinical signs of this disease, the urine is light yellow or yellow, transparent, but a biochemical study revealed the presence of hemoglobin. On the second or third day, after the beginning of the clinical manifestation of the disease, the urine of most animals acquired a red-brown color. When taking blood from most dogs, with a super-acute course of babesiosis, it was more liquid than usual and had a light red color. The number of red blood cells and hemoglobin decreased by one and a half to two times compared to the norm. Peripheral blood smears stained by the Romanovsky-Gimza method and with hematoxylin and eosin showed anisocytosis and poikilocytosis. In red blood cells 
of sick males, round or pear-shaped babesias were found with a size of 3-4 microns (Fig.1). In individual animals, the red blood cells affected by babesias were glued together, forming conglomerates that included from 2 to 15 red blood cells (Fig. 2).

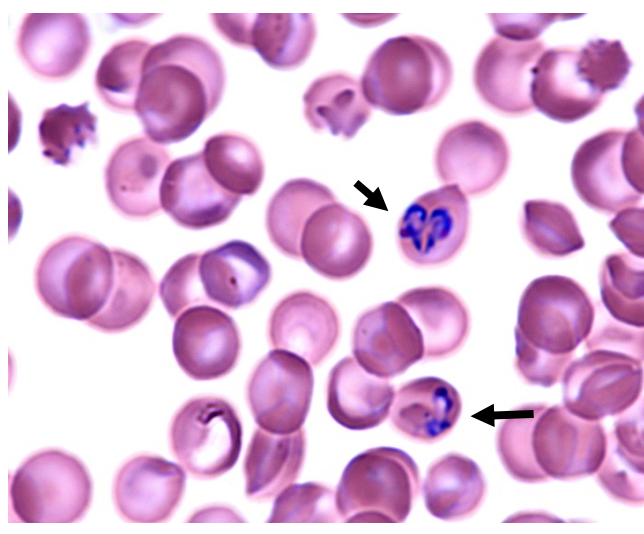

Fig. 1. Babesia in red blood cells (indicated by arrows) in a blood smear of a Pomeranian male, age 5 years, with an acute course of babesiosis. Staining by the Romanovsky-Gimze method $\times 900$.

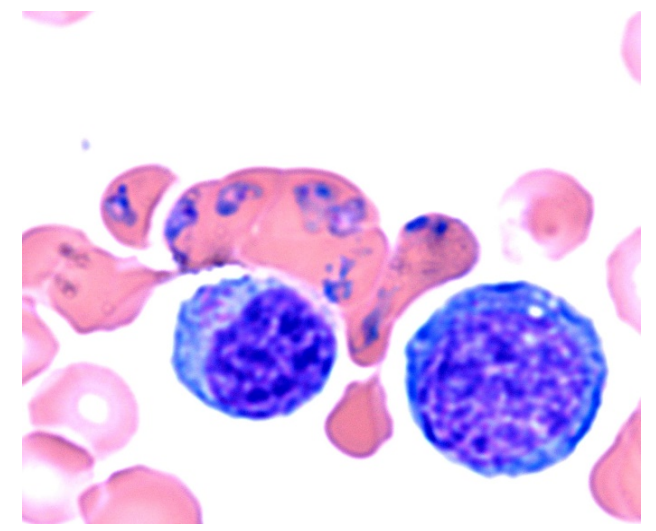

Fig. 2. Bonding of red blood cells affected by babesias. Blood smear of a German shepherd male, age 2 years, with chronic babesiosis. Staining with hematoxylin and eosin $\times 1000$.

When pathoanatomic examination of male cadavers who fell from the ultra-acute and acute course of babesiosis in the external mucous membranes (conjunctiva, mucosa of the oral and nasal cavities), anemia was observed. They were white with a faint pink tinge, and the surface was smooth and wet. Yellowness of the external mucous membranes was not observed in hyperacute cases of the disease. In the acute course of the disease, jaundice was observed only in one of the 6 studied cadavers. In all lymph nodes in the hyperacute course, pathological changes were typical for serous-hemorrhagic lymphadenitis, and in the acute and chronic course for hemorrhagic lymphadenitis. All the studied cadavers had atrophy of the thymus, red bone marrow, hemorrhagic diathesis, protein dystrophy in the liver, kidneys, myocardium, and congestive edema of the lungs and brain. In the gastrointestinal tract, pathoanatomic changes were typical for acute mucosal-catarrhal gastroenteritis in hyperacute and acute disease, and in subacute and chronic disease for subacute and chronic catarrhal gastroenteritis, respectively. No macroscopic changes were found in the testes. The testes were oval in shape, elastic consistency, and cream-colored parenchyma. The lobed structure is not broken. Pathomorphological changes in the testes of all sexually mature males were of the same type.

In the case of hyperacute and acute flow in arterial blood vessels, the changes were typical for alternative arteritis. The endothelium of the arteries is partially desquamated, and the connective tissue fibers of the artery walls are sometimes de-fibrated and homogenized. Focal cell infiltrates consisting of macrophages and lymphocytes, fluid accumulation, and red blood cells were visible in the tissue surrounding arterial vessels (Fig. 3,4). Endocrine cells located between the convoluted seminiferous tubules were in a state of vacuolar degeneration. Part of the endocrine cells were necrotic, and only shadows and vacuoles filled with liquid were visible in their place. In places with large accumulations of fluid, most of the endocrine cells were necrotic (Fig. 5). 


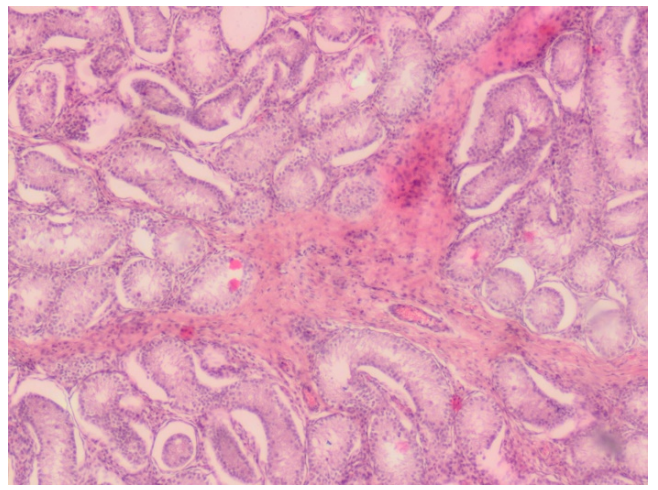

Fig. 3. Accumulation of lymphocyte and macrophage fluid around the artery in the testes of a Pomeranian male, age 5 years, with acute babesiosis. Staining with hematoxylin and eosin $\times 75$.

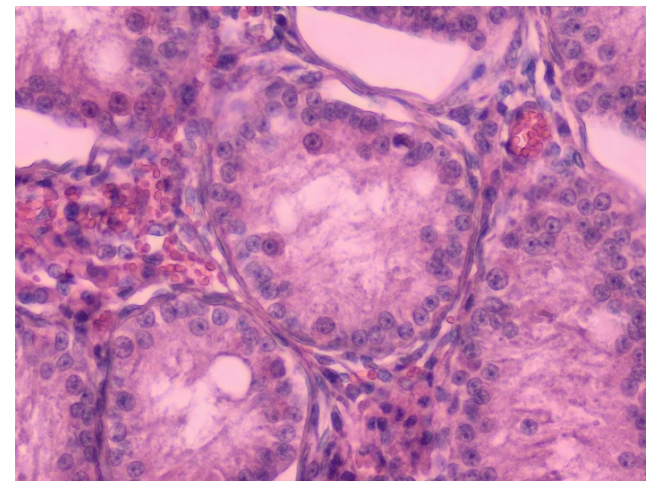

Fig. 4. Focal clusters of red blood cells, lymphocytes and macrophages around the artery in the testes of a Pomeranian male, age 5 years, with an acute course of babesiosis. Staining with hematoxylin and eosin $\times 250$.

These pathomorphological changes in the testes during acute and superacute babesiosis were observed in all the animals studied. The spermatogenic epithelium in $100.0 \%$ of the convoluted seminal tubules was desquamated. In most convoluted seminal tubules, the spermatogenic epithelium did not exceed 2 layers of cells (spermatogonies and first-order spermatocytes). In $40-50 \%$ of the convoluted seminal tubules, the spermatogenic epithelium was completely drained down to the basal plate. The lumen of the convoluted seminal tubules was filled with protein detritus, exfoliated cells of the spermatogenic epithelium. Macrophages are visible in the lumen of the convoluted seed tubules (Fig.6), phaging damaged cells of the spermatogenic epithelium. Mature sperms in the lumen of all the convoluted seminal tubules were completely absent. Part of the Sertoli cells was in a state of vacuolar degeneration, some of them were is necrotic.

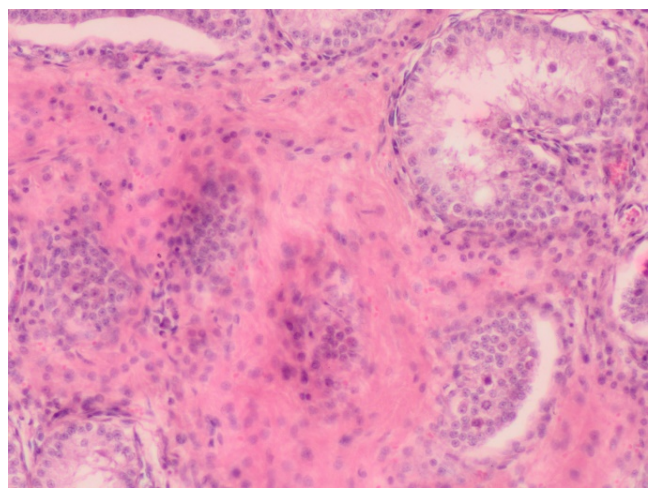

Fig. 5. Extensive accumulation of fluid in interstitial tissue in a Miniature Poodle male, age 1 year, with a super-acute course of babesiosis. Staining with hematoxylin and eosin $\times 150$.

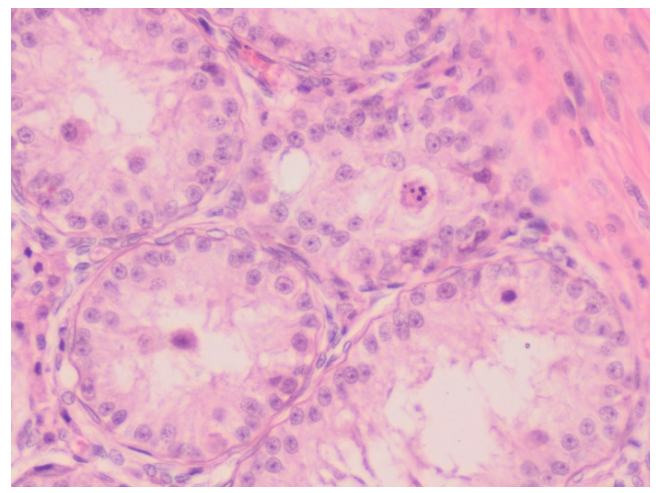

Fig. 6. Desquamation of spermatogenic epithelium and macrophages in the lumen of the tubules in a Pomeranian male, age 5 years, with an acute course of babesiosis. Staining with hematoxylin and eosin $\times 250$.

Microscopy of histological sections of the appendages of the testes in all studied males revealed focal congestive hyperemia in interstitial tissue. In the interstitial tissue surrounding arterial vessels, focal cell infiltrates consisting of macrophages and lymphocytes, and fluid accumulation were visible. The epithelium of the tubules was unchanged, uniform in thickness. In the lumen of the tubules were visible sloughed 
epithelial cells (Fig.7). Mature sperms in the lumen of the tubules of the appendages of the testes were absent.

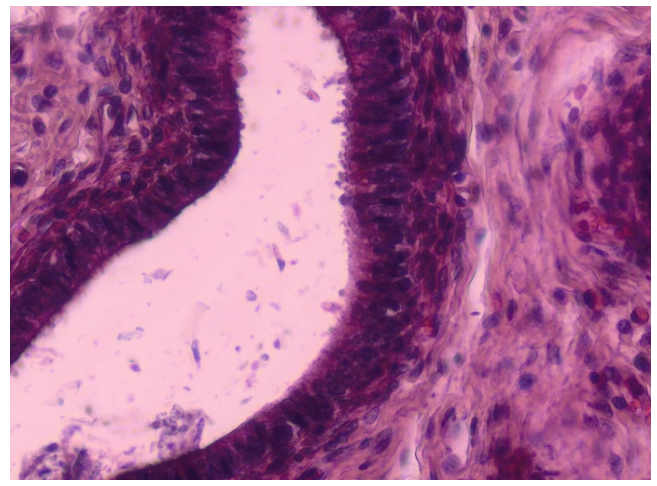

Fig. 7. Exfoliated cells of the spermatogenic epithelium in the lumen of the tubules of the epididymis in a Miniature poodle male, age 2 years, with an acute course of babesiosis. Staining with hematoxylin and eosin $\times 400$.

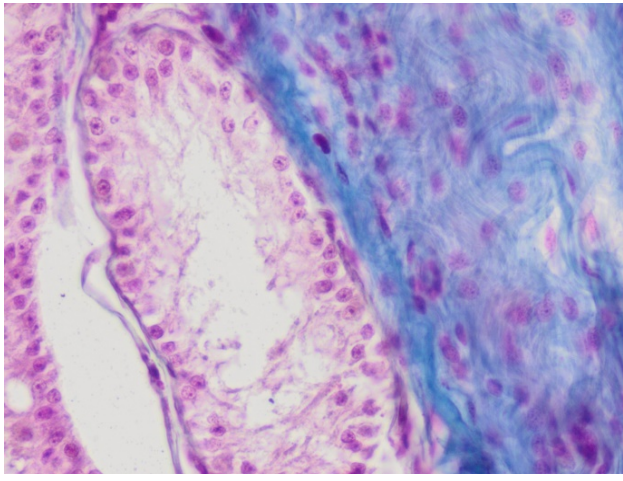

Fig. 8. Desquamation of spermatogenic epithelium in the lumen of the tubules of a German shepherd dog, age 2 years, with chronic babesiosis. Staining by the Mallory method $\times 400$.

In the subacute and chronic course of babesiosis, pathomorphological changes in the epithelium of the convoluted seminal tubules were similar to those in the acute and superacute course and were recorded in all the studied animals. The spermatogenic epithelium in $100.0 \%$ of the convoluted seminal tubules was desquamated and did not exceed the thickness of 2 layers of cells (spermatogonies and first-order spermatocytes) (Fig. 8). In $40-50 \%$ of the convoluted seminal tubules, the spermatogenic epithelium was completely drained down to the basal plate. The lumen of the convoluted seminal tubules was filled with protein detritus, exfoliated cells of the spermatogenic epithelium. In the lumen of the convoluted seminal tubules, macrophages can be seen forming damaged cells of the spermatogenic epithelium. Mature sperms in the lumen of all the convoluted seminal tubules were completely absent.

In interstitial tissue in the subacute course of the disease, cellular infiltrates consisting of macrophages, lymphocytes, a large number of fibroblasts and fibrocytes were visible. In the chronic course of babesiosis, an uneven growth of connective tissue was observed around the convoluted seminal tubules in addition to cellular infiltrates (Fig.9). Especially the plural number of connective tissue elements was seen around the blood vessels. In places of large clusters of connective tissue fibers, endocrine cells were visible in the form of small Islands, the size of which did not exceed 10-15 cells. Some of these cells were reduced in volume compared to the adjacent endocrine cells (Fig.10). 


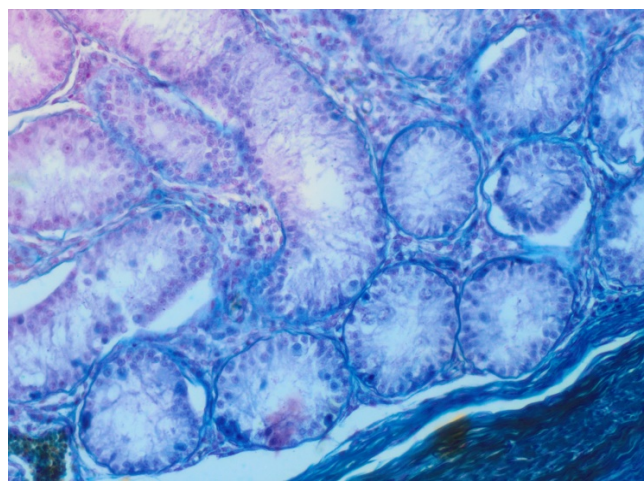

Fig. 9. The growth of connective tissue between the convoluted seminal tubules in a German shepherd male, age 2 years, with chronic babesiosis. Staining by the Mallory method $\times 150$.

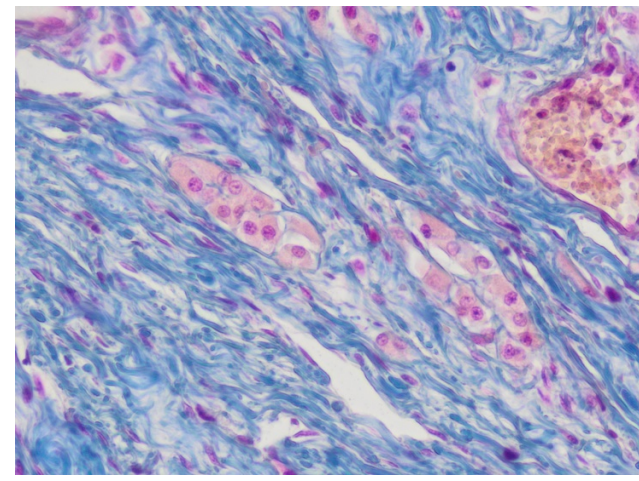

Fig. 10. Atrophy of endocrine cells and growth of connective tissue between the convoluted seminal tubules in a German shepherd dog, age 2 years, with chronic babesiosis. Staining by the Mallory method $\times 150$.

Many authors note that the destruction of the hematotesticular barrier leads to the development of autoimmune orchitis $[10,11,12]$. Autoimmune orchitis is characterized by a decrease in sexual activity, a decrease in the volume of ejaculate and the number of mature sperms in it. Microscopy of sperm smears reveals a large number of pathological forms of sperm. According to many researchers, autoimmune orchitis is characterized by: desquamation of spermatogenic epithelium, phaging by macrophages of sloughed epithelial cells, focal cell infiltration by macrophages, lymphoid, and plasma cells of interstitial tissue. All of the above changes were found in males with babesiosis.

\section{Conclusions}

1. In mature males with acute babesiosis in the testes, pathomorphological changes are typical for acute parenchymal orchitis, characterized by desquamation of the spermatogenic epithelium of the convoluted seminal tubules, phagocytosis by macrophages and giant cells of desquamated cells of the spermatogenic epithelium, as well as dystrophic and necrotic processes in endocrine cells.

2. In subacute and chronic cases of babesiosis in males, pathomorphological changes in the spermatogenic epithelium were similar to those in acute and superacute course, but in the interstitial tissue, in addition, there was a proliferation of connective tissue that compresses endocrine cells that are subject to atrophy.

\section{Acknowledgments}

Based on the results obtained, it can be concluded that in males with babesiosis pathomorphological changes in the testes are characteristic of autoimmune orchitis, which developed as a result of the destruction of the hematotesticular barrier.

\section{References}

1. A. Chauvin, E. Moreau, S. Bonnet, O. Plantard, L. Malandrin, Babesia and its hosts: Adaptation to long-lasting interactions as a way to achieve efficient transmission 40, 37 (2009) DOI: 10.1051/vetres/2009020 
2. A. Swei, K.E. O'Connor, L.I. Couper, J. Thekkiniath, and others, Dermacentor albipictus 49, 95-103 (2019), DOI: 10.1016/j.ijpara.2018.07.002

3. J. Gonzalez, I. Echaide, A. Pabón, J. Gabriel Piñeros, S. Blair, A. Tobón-Castaño, Babesiosis prevalence in malaria-endemic regions of Colombia 55, 222-229 (2018) DOI: 10.4103/0972-9062.249480

4. F. Jongejan, B.-L. Su, H.-J. Yang, L. Berger, and others, A novel vector for canine babesiosis 11, 134 (2018) DOI: 10.1186/s13071-018-2722-y

5. I. Baráková, M. Derdáková, D. Selyemová, M. Chvostáč, E. Špitalská, F. Rosso, M. Collini, R. Rosà, Tick-borne pathogens and their reservoir hosts in northern Italy $\mathbf{9}$, 164-170 (2018) DOI: 10.1016/j.ttbdis.2017.08.012

6. F. Bousmaha, Khoudja, F. Benchaib, Asian Journal of Animal And Veterinary Advances 7(10), 950-959 (2012) doi 10/3923//ajava/2012/950/959

7. H.C. Schuppe, A. Pilatz, H. Hossain, A. Meinhardt, M. Bergmann, G. Haidl, W. Weidner, Researcher ID ORCID UROLOGE 49, 629-635 (2010) doi: 10.1007/s00120010-2256-1

8. C.A. Silva, M. Cocuzza, J.F. Carvalho, E. Bonfa, Autoimmunity Reviews 13(4-5), 431-434 (2014) doi: 10.1016/j.autrev.2014.01.024

9. V.I. Trukhachev, V.V. Rodin, V.V. Mikhailenko, A.A. Dergunov, Chemical Abstracts 147, 253438 (2007)

10. M. Naito Terayama, H. Hayato, S. Hirai et al., Medical Molecular Morphology 45(4), 185-189 (2012) DOI: 10.1007/s00795-012-0587

11. C. Matschurat, K. Rode, J Hollenbach, K. Wolf, C. Urhausen, A. Beineke, A.R. Gunzel-Apel, R. Brehm, Histology and Histopathology 34(5), 525-535 (2019) doi: 10.14670/HH-18-058

12. H.-C. Schuppe, A. Pilatz, H. Hossain, A. Meinhardt, M. Bergmann, G. Haidl, W. Weidner, Urologe 49(5), 629-635 (2010) doi: 10.1007/s00120-010-2256-1 